\title{
Childhood acute generalized exanthematous pustulosis
}

\section{Masato Ishikawa, Tatsuhiko Mori, Yuka Hanami, Toshiyuki Yamamoto}

\author{
Department of Dermatology, Fukushima Medical University, Fukushima, Japan
}

Corresponding author: Dr. Masato Ishikawa, E-mail: ishimasa@fmu.ac.jp

Sir,

Acute generalized exanthematous pustulosis (AGEP) is an uncommon disease which is usually caused by systemic medication, and its incidence is reported to be $0.35-5$ per million per year $[1,2]$. It is especially rare for AGEP to develop on childhood. We herein describe a pediatric case of AGEP induced by ampicillin.

A 13-year-old boy was hospitalized to the Plastic Surgery Department in our hospital for surgical treatment of scar contracture after extensive burns. He was referred to our department, complaining of high fever and skin rash. In the perioperative period, he was administered ampicillin, cefaclor, non-steroidal anti-inflammatory drugs, acetaminophen, and rabeprazole sodium. Three days after from surgery, itchy erythema appeared to his back. As a result of having been given ampicillin again due to fever, the skin rash spread to a whole body rapidly. Physical examination revealed erythroderma with a number of pinpoint pustules (Figs. la and b). Symptoms such as hyperemia were not seen in the mucous membrane, and Nikolsky sign was negative. Laboratory data showed elevated white blood cell count (17600/ $\mu$ l, with 81\% neutrophils), and C-reactive protein $(6.36 \mathrm{mg} / \mathrm{dl})$. Although a little staphylococcuses were detected in pustule, no bacteria were detected in the blood. A skin biopsy showed pustules in the epidermis with spongiform pustules, and perivascular inflammatory cell infiltration, mainly composed of lymphocytes and eosinophils, in the upper dermis (Fig. 2). After discontinuation of ampicillin and initiation of oral prednisolone $(25 \mathrm{mg} / \mathrm{day})$, the erythema and fever quickly disappeared. Patch test showed positive reaction to $10 \%$ and $20 \%$ ampicillin and negative reaction to all the other drugs used in the perioperative period.

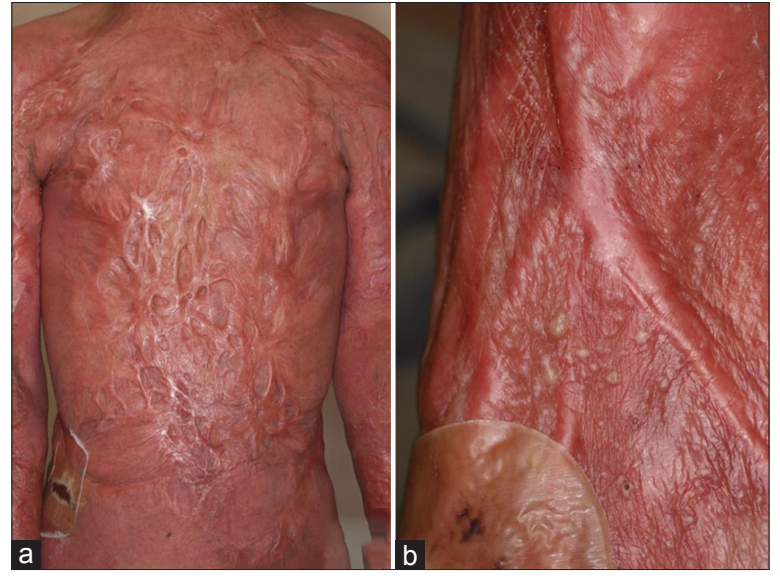

Figure 1: (a) Clinical feature on the trunk showing erythroderma with a number of pinpoint pustules. (b) Pinpoint pustules on the back of the hand.

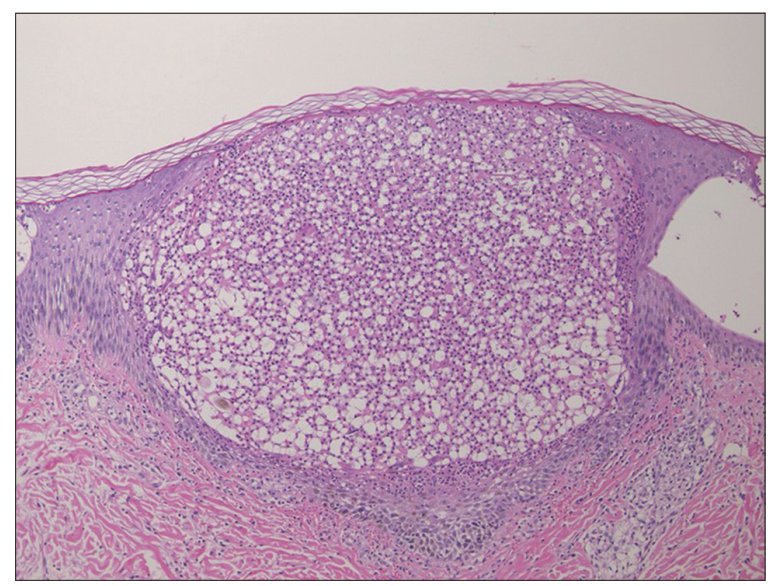

Figure 2: Histopathological specimen showed spongiform pustules in the epidermis, and perivascular inflammatory cell infiltration, mainly composed of lymphocytes and eosinophils, in the upper dermis (hematoxylin-eosin, original magnification x100).

To date, only six English literature cases of childhood AGEP have been reported. The age of patients were

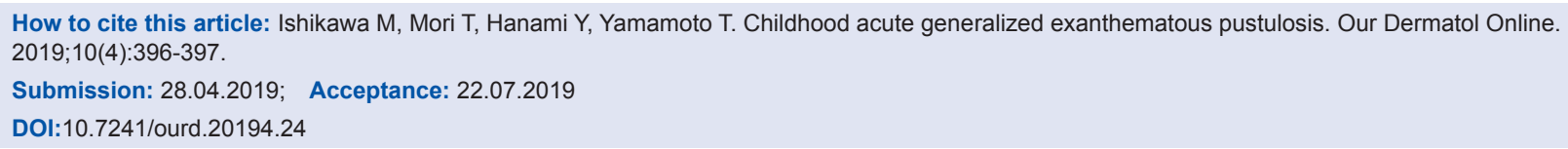


17 months [3], 4-year-old, 7-year-old, 11-year-old, 12-year-old [4], 13-year-old [5], and 14-year-old (our case). It was assumed that the skin rash was caused by amoxicillin [3], contrast agent, paracetamol, cetirizine, ketoconazole [4], and amoxicillin-clavulanic acid [5]. One case was relieved by just ceasing the application of the suspected drug [3], and other cases were needed another antihistaminic drug, corticosteroid ointment, antihistaminic drug [5], and intravenous corticosteroid $[4,5]$. In our case, the patient showed erythroderma, and we needed oral prednisolone. To date, there are no reports of such the serious case of childhood AGEP.

The main differentiation diagnosis is that of generalized pustular psoriasis (GPP). It is often difficult to distinguish both diseases by eruption and histopathology. It is necessary to pay attention to recurrence in long-term clinical progress to distinguish between both diseases, as AGEP only rarely recurs, but GPP recurs many times. In pediatric cases, the differentiation from Kawasaki disease is also necessary, because Kawasaki disease rarely presents with skin rash similar to AGEP, which manifests as an erythematous pustular eruption. Although rare, we should bear in mind that AGEP can occur even in children.

\section{Consent}

The examination of the patient was conducted according to the Declaration of Helsinki principles.

\section{REFERENCES}

1. Sidoroff A, Halevy S, Bavinck JN, Vaillant L, Roujeau JC. Acute generalized exanthematous pustulosis (AGEP): a clinical reaction pattern. J Cutan Pathol. 2001;28:113-9.

2. Davidovici B, Dodiuk-Gad R, Rozenman D, Halevy S; Israeli RegiSCAR Network. Profile of acute generalized exanthematous pustulosis in Israel during 2002-2005: results of the RegiSCAR Study. Isr Med Assoc J. 2008;10:410-2.

3. Meadows KP, Egan CA, Vanderhooft S. Acute generalized exanthematous pustulosis (AGEP), an uncommon condition in children: case report and review of the literature. Pediatr Dermatol. 2000;17:399-402.

4. Miteva L, Kadurina M, Schwartz RA. Childhood acute generalized exanthematous pustulosis induced by oral ketoconazole. Acta Dermatovenerol Croat. 2010;18:267-70.

5. Riten K, Shahina Q, Jeannette J, Palma-Diaz MF. A severe case of acute generalized exanthematous pustulosis (AGEP) in a child after the administration of amoxicillin-clavulanic acid: brief report. Pediatr Dermatol. 2009;26:623-5.

Copyright by Masato Ishikawa, et al. This is an open-access article distributed under the terms of the Creative Commons Attribution License, which permits unrestricted use, distribution, and reproduction in any medium, provided the original author and source are credited.

Source of Support: Nil, Conflict of Interest: None declared. 